\title{
PAUTAS DE DISEÑO PARA DISMINUIR LAS TEMPERATURAS URBANAS EN REGIONES CON ALTA PRODUCTIVIDAD SOLAR. PARÁMETROS MORFOLÓGICOS Y MATERIALES.
}

\section{DESIGN GUIDELINES TO REDUCE URBAN TEMPERATURES IN REGIONS WITH HIGH SOLAR PRODUCTIVITY: MORPHOLOGICAL AND MATERIALS PARAMETERS.}

\author{
NOELIA LILIANA ALCHAPAR \\ Instituto de Ambiente, Hábitat y Energía (INAHE). CONICET. \\ Centro Científico y Tecnológico de Mendoza. CCT-Mendoza. \\ Mendoza, Argentina \\ nalchapar@mendoza-conicet.gob.ar \\ ERICA NORMA CORREA \\ Instituto de Ambiente, Hábitat y Energía (INAHE). CONICET. \\ Centro Científico y Tecnológico de Mendoza. CCT-Mendoza. \\ Mendoza, Argentina \\ ecorrea@mendoza-conicet.gob.ar
}

\begin{abstract}
RESUMEN
El estudio aquí expuesto examinó el potencial de las características del perfil urbano -morfología y materialidad- para disminuir las temperaturas urbanas. El objetivo consistió en identificar pautas de diseño y rehabilitación urbana, que permitan densificar áreas residenciales de la ciudad de Mendoza, Argentina. Para ello fueron simulados 32 escenarios con el programa ENVI-met 3.1, en los cuales se combinó distintos niveles de reflectancia solar (albedo) de los materiales que conforman las envolventes, diversas morfologías de perfil urbano, y presencia y ausencia de forestación dentro del canal vial, variando su orientación E-O y N-S. A partir de los resultados obtenidos por las simulaciones, se infiere que aquellos canales viales que presentan un comportamiento térmico más eficiente, están vegetados, y, morfológicamente, su perfil urbano es continuo sobre la línea municipal. En cuanto a la materialidad de sus envolventes, presentan niveles de albedo altos (0.7) en techos y pavimentos, y niveles de albedo bajo (0.3) en fachadas.
\end{abstract}

Palabras clave

diseño urbano, fachadas, materiales, morfología.

\begin{abstract}
This study examined the numerical modeling performance of morphological and materials alternatives for reducing urban temperatures. The aim was to identify design and urban regeneration guidelines that enable the densification of residential areas in the city of Mendoza, Argentina. To this end, using ENVI-met 3.1 software 32, scenarios were simulated that combined: different levels of solar reflectance (albedo) of building envelope materials, different urban profile morphologies, the presence or absence of forestation in urban canyons, and E-W/N-S variation in orientation. From the simulation results it can be inferred that there is more efficient thermal performance in the vegetated scenarios that morphologically have a continuous urban profile above the municipal building line. Regarding envelope materiality, there are high albedo levels (0.7) on roofs and pavements, and low albedo levels (0.3) on façades.
\end{abstract}

Keywords urban design, façades, materials, morphology. 


\section{INTRODUCCIÓN.}

En virtud de los desafíos de la creciente urbanización y el cambio climático actual, hay una necesidad urgente de desarrollar estrategias de adaptación/mitigación sostenible en el diseño y planificación de las ciudades. Se pueden citar numerosas razones por las que considerar la capacidad para evaluar y diseñar barrios urbanos adaptados al cambio climático resulta importante.

Uno de los objetivos fundamentales de la planificación urbana y diseño barrial es ofrecer un entorno exterior favorable a los habitantes, minimizando el consumo energético edilicio y manteniendo los rangos de confort térmico interior y la calidad del aire exterior. En muchos aspectos, el comportamiento medioambiental de un barrio urbano establece las condiciones del rendimiento ambiental y energético de los edificios individuales situados dentro de éste (Thorsson et al., 2010).

La habitabilidad de los espacios exteriores está influenciada por el microclima urbano, pero aunque los efectos de los diversos parámetrosde diseño -morfología, materialidad, orientación, vegetación, etc.- dentro de un entorno urbano han sido ampliamente evaluados y documentados (Givoni et al., 2003; Santamouris, 2007), continúan siendo limitados aquellos estudios que abordan el rol de cada uno de estos parámetros sobre el clima urbano (Shashua-Bar, Tsiros y Hoffman, 2012).

Los escenarios urbanos presentan una estrecha relación entre su comportamiento térmico y la selección de materiales y morfología urbano/edilicia. Mientras los materiales incrementan la permeabilidad y la capacidad calorífica de las superficies, la morfología edilicia contribuye con la captura de radiación "radiative trapping" en un recinto urbano (Doya, Bozonnet y Allard, 2012; Correa, 2006).

La selección de los materiales de la envolvente constituye un parámetro que debe ser analizado en cada caso particular, pues el incremento de los niveles de reflectancia solar -también denominado albedo- mejora las condiciones térmicas del medio ambiente, especialmente en cañones vegetados (Akbari, Menon y Rosenfeld, 2009; Santamouris, 2014). Ahora bien, Erell et al. (2014) informó que si bien el uso extensivo de materiales con alto albedo en cañones urbanos - de regiones de clima cálido- disminuye las temperaturas del aire, puede incrementar notablemente la carga de radiación solar, comprometiendo el confort térmico peatonal. Esto también fue enunciado en estudios desarrollados por Yaghoobian y Kleissl (2012), quienes destacaron que el aumento de los niveles de albedo en pavimentos, desde 0.1 a 0.5 , incrementa las cargas de enfriamiento anuales hasta en un $11 \%$, en un edificio de oficinas en Phoenix, Arizona.
En lo referente a la morfología urbana/edilicia, estudios desarrollados por Ali-Toudert y Meyer (2006), y Johansson y Emmanuel (2006) se enfocaron en los efectos de la geometría y la orientación en cañones urbanos en ciudades cálidas y húmedas. Pearlmutter, Berliner y Shaviv (2007) analizaron también la relación entre la densidad urbana y la habitabilidad en espacios exteriores en una región árida de Israel. Estas investigaciones encontraron una correlación directa entre la eficiencia térmica de un recinto urbano y su morfología. Los resultados muestran patrones contrastantes de confort térmico entre calles urbanas profundas y superficiales, así como entre las distintas orientaciones estudiadas.

En este marco, el presente estudio proyecta producir conocimiento en torno a desarrollar lineamientos de diseño para conseguir el máximo nivel de densificación (18 metros, según Código Municipal) de un sector residencial ubicado en la ciudad de Mendoza, Argentina, con el mínimo impacto térmico y ambiental. Para ello, se evaluaron dos tipologías de perfiles urbanos, distintos niveles de reflectancia solar (albedo) de la materialidad de las envolventes urbano-edilicias, el efecto de la forestación en dos situaciones, cañón urbano vegetado y sin vegetar, y orientación E-O y N-S.

Un cañón urbano está definido por dos parámetros principales: la altura máxima de los edificios $(\mathrm{H})$ y el ancho comprendido entre líneas de edificación (W); el cociente de ambos valores determina el aspecto de ratio $\mathrm{H} / \mathrm{W}$.

La presente investigación es de carácter inicial, sin embargo, propone dar pautas de diseño urbano-edilicias preliminares para la elaboración de ordenanzas municipales en ciudades con alta disponibilidad de radiación solar. A fin de concretar en el corto y mediano plazo un crecimiento metropolitano más eficiente desde el punto de vista energético y ambiental en ciudades emplazadas en contextos áridos.

\section{METODOLOGÍA.}

La presente investigación se llevó a cabo en el área Metropolitana de Mendoza localizada en el centro oeste de Argentina. Según clasificación de Köppen, el clima de la ciudad es desértico, tipo BWk (Kottek et al., 2006). Registra $218 \mathrm{~mm}$ de precipitación anual, 56 \% de humedad relativa de aire anual, $1022 \mathrm{~W} / \mathrm{m}^{2}$ de radiación solar diaria máxima promedio en verano, $1.7 \mathrm{~m} / \mathrm{s}$ de velocidad de viento anual $(\mathrm{h}=10 \mathrm{~m})$ en dirección predominante del sudeste. La temperatura de aire media anual es de $16.50^{\circ} \mathrm{C}$, con máxima media de $24.50{ }^{\circ} \mathrm{C}$ y mínima media de $9.60{ }^{\circ} \mathrm{C}$ (Observatorio de Mendoza). 


\begin{abstract}
ALBEDO BAJO
ALBEDO ALTO

(â promedio $=0.3$ )

(â promedio $=0.7$ )

- Pavimentos peatonales con â $\leq 0.30$. Pavimentos peatonales cementicios (P27, P35, P07).

- Fachadas con $\hat{a} \leq 0.35$. Revestimientos texturados acrílicos (SIP 40, SIP 21, SIP 15, SIP 12, $\mathrm{CW}$ 64); y pinturas para exteriores (PIN 02, PIN 16).

- Techos con â $\leq 0.40$. Tejas (T13, T12). (Ver Figura 1.a)

- Pavimentos peatonales con â $\leq$ 0.70. Pavimentos
peatonales (P13, P25, P34).
- Fachadas con â $\leq$ 0.80. Revestimientos
texturados (SIP 22, SIP 18, SIP 03, SIP 27, SIP 17,
SIP 09, SIP 10, SIP 01, SIP 34, SIP 02, SIP 33, SIP
26, SIP 25, CW 53, CW 60, CW 46, CW 67, CW 59,
CW 78, CW 75, CW 70, CW 54, CW 62, CW 66,
CW 74, CW 43, CW 51, CW 58, CW 73, CW 42,
CW 57, CW 50, CW 65, CW 41, CW 49); pinturas
exteriores (PIN 08, PIN 01, PIN 11, PIN 12, PIN 09,
PIN 10, PIN 03, PIN 06, PIN 04, PIN 07, PIN 14,
PIN 05, PIN 15, PIN 13).
- Techos con â $\leq 0.70$. Tejas (T14, T01, T03, T08).
(Ver Figura 1.b)
\end{abstract}

Nomenclaturas: P: Pavimentos peatonales; SIP: Revestimientos texturados acrílicos, CW: Revestimientos texturados, cementicios; T: Tejas; PIN: Pinturas para exterior.

Tabla 1. Clasificación de materiales evaluados según rangos de albedo (â). Fuente: Elaborada por el autor.

El proceso metodológico incluyó cuatro etapas:

(1) caracterización de las propiedades ópticas y comportamiento térmico de materiales de la envolvente urbana; (2) selección y monitoreo del área a intervenir; (3) modelación numérica con el programa ENVI-met 3.1 del área; (4) simulación de 32 escenarios de rehabilitación, que combinan rangos de albedo de los materiales de la envolvente urbana y perfiles urbanos, según porcentaje de vegetación y orientación.

Resulta importante resaltar que esta metodología puede ser replicada en otras zonas climáticas y contextos socioeconómicos, en busca de la regionalización de pautas de diseño urbana.

\section{CARACTERIZACIÓN ÓPTICA DE MATERIALES DE LA ENVOLVENTE URBANA.}

Con el fin de obtener indicadores respecto a la habilidad de los materiales para reducir las temperaturas de aire exteriores, se ensayaron materiales de la envolvente urbana de mayor difusión en el mercado local. La muestra está compuesta por 152 materiales: tejas, membranas, revestimientos texturados, pinturas para fachadas y pavimentos peatonales. Para cuantificar la capacidad reflectiva (albedo) y el comportamiento térmico superficial de los materiales, se utilizó un método variante de la norma ASTME-1918 propuesto por Akbari, Levinson y Stern (2008). Dicho método mide la reflectancia solar de un material sobre $1 \mathrm{~m}^{2}$ de superficie, mediante el uso de un piranómetro y un par de máscaras de color blanco y negro. Los resultados han sido publicados en diversos trabajos nacionales e internacionales (Alchapar, Correa y Cantón, 2014; Alchapar y Correa, 2015).

En esta investigación se detallan aquellos materiales que registran rangos de albedo extremos, es decir, albedo bajo con nivel de reflectancia solar inferior a 0.3 , y albedo alto correspondiente a un nivel de reflectancia solar superior a 0.7; ambos detallados en la Tabla 1 y la Figura 1. 

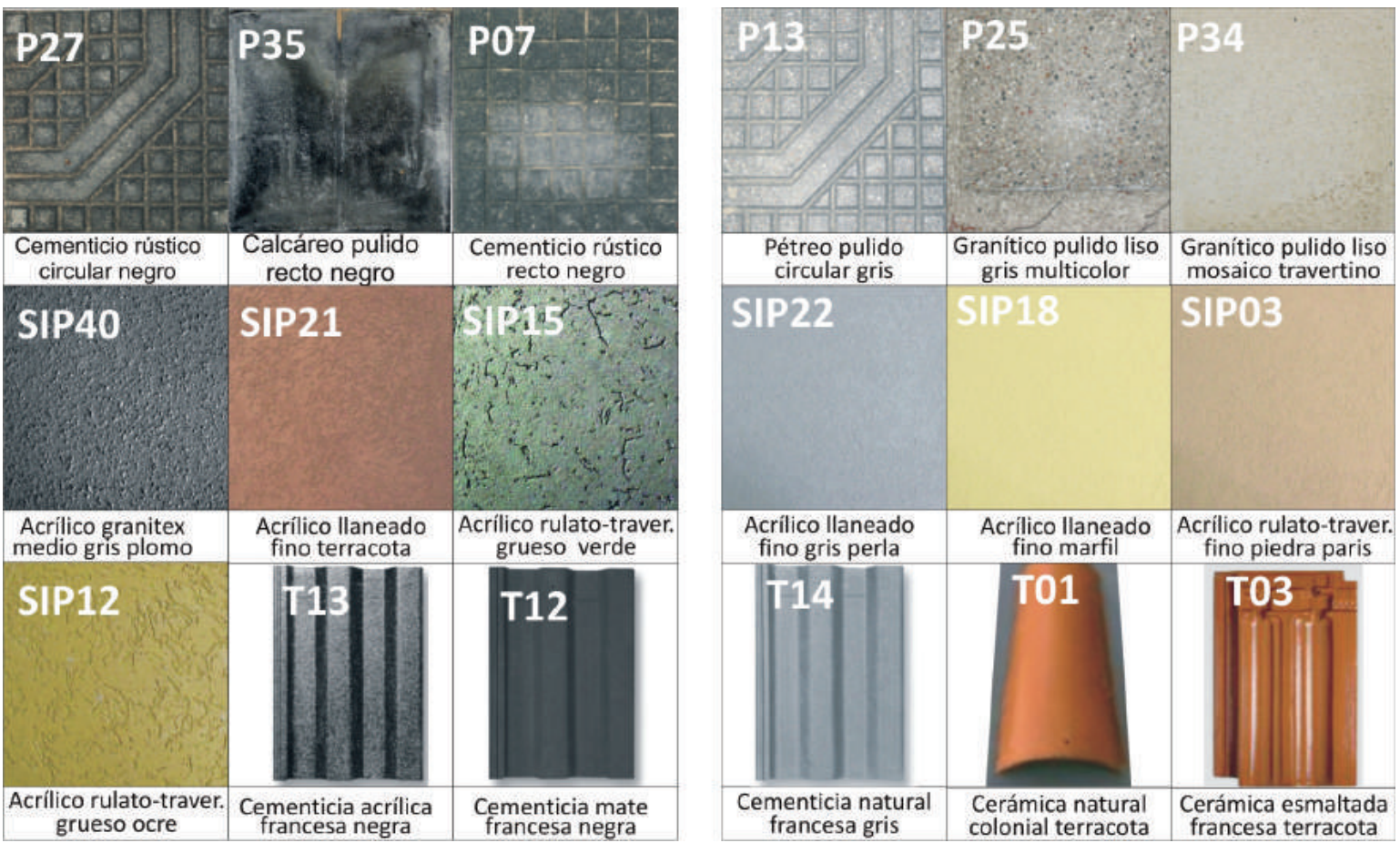

Figura 1. Detalle de materiales evaluados según nivel de albedo. Pavimentos peatonales (P), fachadas (SIP) y techos (T). Se grafican los tres primeros materiales para cada rango. Materiales con albedo bajo $=0.3$ (izquierda) y materiales con albedo alto=0.7. Fuente: Elaboración del autor.

\section{SELECCIÓN Y MONITOREO DEL CASO DE ESTUDIO.}

El sector a intervenir tiene una superficie $210 \times 210 \mathrm{~m}(4.4$ hectáreas) y comprende las calles: E-W -Pasteur, Dr. Lemos y Tropero Sosa-; N-S - Roque Sáenz Peña y San Martín(32 $\left.54^{\prime} 47^{\prime \prime} S, 68^{\circ} 50^{\prime} 46^{\prime \prime} \mathrm{O}\right)$. El área tiene una configuración ortogonal, con una altura edilicia entre 1 y 3 niveles, y el aspecto de ratio es igual a $\mathrm{H} / \mathrm{W}=0.3$ a 0.4 . Presenta un $60 \%$ de forestación urbana, principalmente con "morera blanca" (Morus alba), que es una especie arbórea de 15 metros de alto y hoja caduca.

Esta zona ofrece grandes posibilidades para su densificación en altura, debido a una serie de factores, como proximidad al centro de la ciudad, localización en relación a los ejes de circulación y disponibilidad de servicios e infraestructura. De acuerdo al Código de Edificación Municipal, y su capítulo UIII "De las Normas de Zonificación e Impacto Ambiental", el área corresponde a una sub-zona residencial, tipo R6. El destino es de uso predominantemente residencial uni y multifamiliar, con funciones complementarias y compatibles. La densidad neta media es de 250 a 400 habitantes por hectárea. Según indicadores urbanos, el factor de ocupación del suelo del área (FOS) es de 0.70 y el factor de ocupación total (FOT) es de 4.20. La altura máxima de construcción permitida en el sector es de 6 niveles, es decir, 18 metros de altura, de acuerdo a lo establecido en Ordenanza N ${ }^{\circ} 5519 / 07$ y sus correspondientes actualizaciones, las Ordenanzas N58352010 y N5924/11
El monitoreo térmico y ambiental se llevó a cabo en la temporada de verano, en días estables sin ocurrencia de precipitaciones. Los datos utilizados para la calibración corresponden al 14 de enero por presentar condiciones estándares al periodo estival de Mendoza. En ese día se registraron las siguientes condiciones: temperatura máxima de $36{ }^{\circ} \mathrm{C}$; temperatura mínima de $21{ }^{\circ} \mathrm{C}$; temperatura promedio de $27{ }^{\circ} \mathrm{C}$; radiación global máxima $1.174 \mathrm{~W} /$ $\mathrm{m}^{2}$; y humedad relativa promedio $25 \%$ (Observatorio de Mendoza).

A fin de calibrar los datos simulados con los medidos, fueron recogidas variables de temperatura ( $\mathrm{Ta}$ ) y humedad relativa (HR) del aire por hora. Para ello, se colocó una estación fija tipo HOBO H08-003-02 a 2.5m de altura dentro del canal vial -Pf-. (Figura 2b). Se tomaron registros de velocidad de viento $(V v)$ desde una estación meteorológica móvil tipo ONSET Weather HOBO H21-001 (Figura 2a). También se recogieron datos de temperatura superficial de suelo (Tsuelo) en el punto Pf durante un periodo de 12:00 hs con un termómetro infrarrojo tipo FLUKE 66 (Figura 2c). 

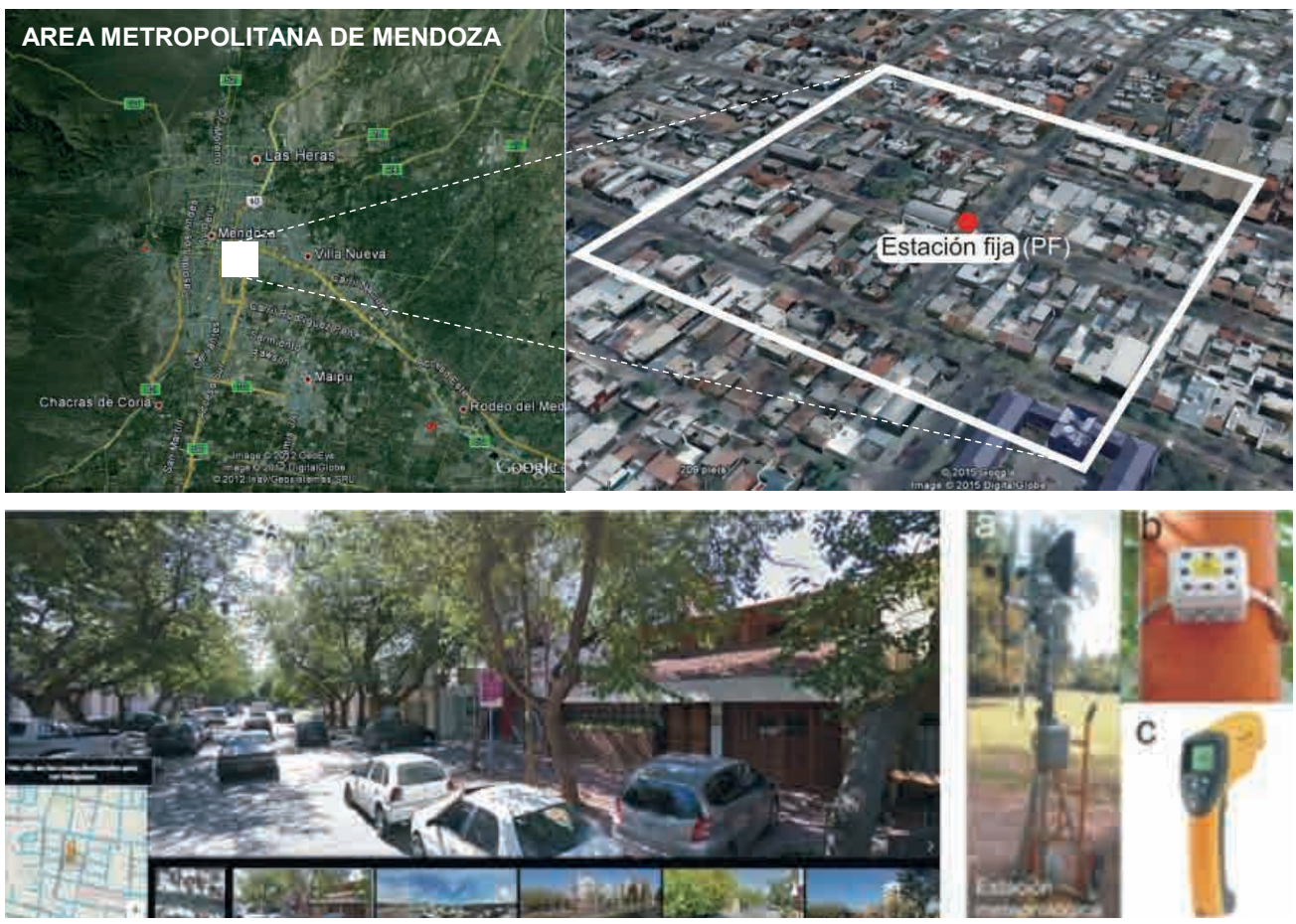

Figura 2. Vista escala satelital del área de estudio y ubicación de la estación fija (Pf) (arriba). Fuente: Google maps, 2015. Vista peatonal del área de estudio y detalle del instrumental utilizado para la colecta de datos. (a) Estación meteorológica móvil tipo ONSET Weather HOBO H21-001; (b) Estación fija -Pf- tipo HOBO H08-003-02; (c) Termómetro infrarrojo tipo FLUKE 66 (abajo).

\section{DISEÑO DEL MODELO NUMÉRICO ENVI-MET 3.1.}

Para realizar el diseño numérico se utilizó el programa de acceso gratuito ENVI-met 3.1, desarrollado por Michael Bruse en el Instituto de Geografía de la Universidad de Mainz, Alemania. Este modelo computacional tridimensional trabaja a escala de microclima urbano y simula las interacciones entre el aire y la superficie del entorno urbano con una resolución típica de 0.5 a 10 metros en el espacio y cada 10 segundos en tiempo.

ENVI-met 3.1 está basado en las leyes fundamentales de la dinámica de fluidos y la termodinámica. El modelo incluye la simulación de: flujos alrededor y entre edificios; procesos de intercambio de calor y vapor de las superficies de suelo y paredes; turbulencia; parámetros de la vegetación; bioclimatología; y dispersión de contaminantes (Bruse, 2006).

\section{VARIABLES DE INGRESO AL SIMULADOR.}

Los datos de ingreso para la modelación numérica del área evaluada se pueden dividir en tres grupos:

- Diseño del espacio físico: El modelo fue realizado en una versión $100 \times 100 \times 30$. La resolución del área es de $3 \times 3 \times 3$ y malla de $70(x) ; 70(y)$, debido a que la superficie de referencia es de $210 \times 210 \mathrm{~m}$.
- Variables climáticas: El software ENVImet 3.1 requiere del ingreso de variables no perturbadas que caracterizan las condiciones de borde de la simulación, tales como: (i) velocidad, dirección de viento $(\mathrm{m} / \mathrm{s})$ a $10 \mathrm{~m}$ de altura y rugosidad de suelo $(\mathrm{z} 0)$ al punto de referencia; (ii) temperatura atmosférica inicial (K) y humedad específica (gr. agua/kg. aire) a 2500 m de altura. Los datos se obtuvieron del Aeropuerto Francisco Gabrielli -Estación $n^{\circ}$ 87418, Observatorio Aero de Mendoza - en colaboración con la Universidad de Wyoming; (iii) humedad relativa (\%) a $2 \mathrm{~m}$ de altura, registrada con sensor ONSET Weather, tipo HOBO H08-003-02 (punto fijo, para el ajuste).

- Propiedades térmicas del modelo teórico: Para la caracterización de los edificios es necesario definir temperatura interior, transmitancia térmica y albedo de paredes, techos y pavimentos. Con el propósito de especificar el comportamiento del suelo, se debe precisar temperatura y humedad para distintas capas de suelo. En la Tabla 2 están listadas las condiciones de simulación y las propiedades usadas en el trabajo. 
Tabla 2. Parámetros de ingreso del simulador ENVI-met 3.1

\begin{tabular}{|c|c|c|}
\hline \multicolumn{3}{|l|}{ DATOS } \\
\hline \multirow{6}{*}{ Principales } & Velocidad de viento a $10 \mathrm{~m}[\mathrm{~m} / \mathrm{s}]$ & 3 \\
\hline & Dirección de viento (0:N; 90:E;180:S; 270:W) & 150 \\
\hline & Rugosidad (z0) al punto de referencia & $0.1^{*}$ \\
\hline & Temperatura atmosférica inicial $[\mathrm{K}]$ & 300 \\
\hline & Humedad específica a 2.500m [g agua/kg aire] & 2.8 \\
\hline & Humedad Relativa a 2m [\%] & 28 \\
\hline \multirow{3}{*}{ Edilicios } & Temperatura interior $[\mathrm{K}]$ & 299 \\
\hline & Transmitancia térmica paredes $\left[\mathrm{W} / \mathrm{m}^{2} \mathrm{~K}\right]$ & 2 \\
\hline & Transmitancia térmica techos $\left[\mathrm{W} / \mathrm{m}^{2} \mathrm{~K}\right]$ & 0.76 \\
\hline \multirow{6}{*}{ De Suelo } & Temperatura Inicial de capa superior $(0-20 \mathrm{~cm}) \quad[\mathrm{K}]$ & 300 \\
\hline & Temperatura Inicial de capa media $(20-50 \mathrm{~cm})[\mathrm{K}]$ & 305 \\
\hline & Temperatura Inicial de capa profunda (más de $50 \mathrm{~cm}$ ) [K] & 305 \\
\hline & Humedad Relativa de capa superior $(0-20 \mathrm{~cm})[\%]$ & $50^{*}$ \\
\hline & Humedad Relativa de capa media $(20-50 \mathrm{~cm})[\%]$ & $60^{*}$ \\
\hline & Humedad Relativa de capa profunda (más de $50 \mathrm{~cm}$ ) & $60 *$ \\
\hline
\end{tabular}

Nota: Los parámetros con "*"son valores por defecto de ENVI-met 3.1.

\section{CALIBRACIÓN DEL MODELO NUMÉRICO ENVI-MET 3.1.}

Para ajustar el modelo numérico se contrastó la curva de temperatura del aire del espacio simulado con ENVI-met (Ps), con la curva temperatura del aire obtenida a partir de datos registrados en un punto fijo de referencia (Pf), ubicado dentro del canal vial analizado e indicado en la Figura 2.

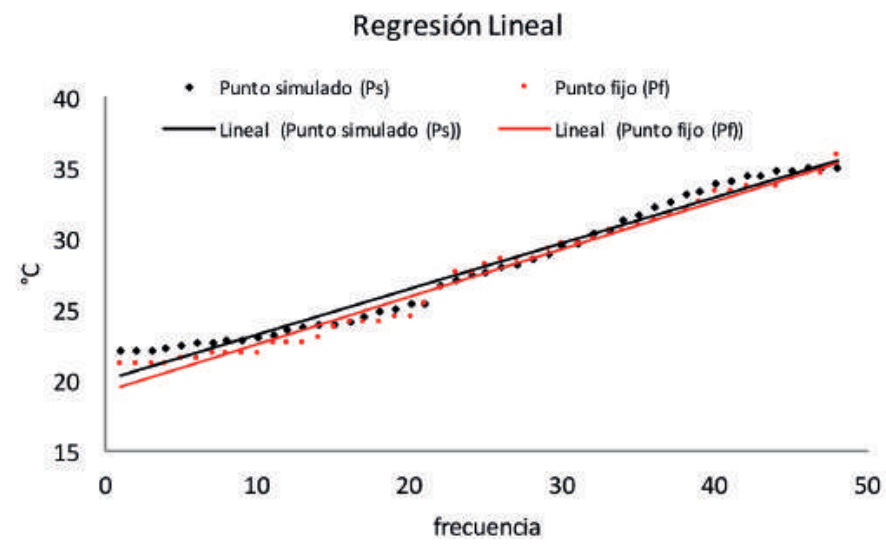

La Figura 3 grafica la regresión lineal de distribución de los datos medidos (Pf) con los simulados (Ps). Los estadísticos que caracterizan y comparan los datos muestran una buena predicción del comportamiento térmico del día evaluado, con un error cuadrático medio $\mathrm{RMSE}=3.13 \%$ y error absoluto medio $\mathrm{MBE}=0.06 \%$.

\section{ESCENARIOS DE DENSIFICACIÓN PROPUESTOS.}

Con el fin de evaluar el grado de impacto de parámetros de diseño urbano sobre las temperaturas exteriores, fueron modelados 32 escenarios que modifican:

Rangos de albedo (â): Se simularon 4 configuraciones de albedo de envolventes. La $1^{\circ}$ y $2^{\circ}$ alternativa corresponden a un albedo homogéneo en todas las superficies de la envolvente urbana (alto y bajo). Las $3^{\circ}$ y $4^{\circ}$ configuraciones corresponden a un albedo combinado, es decir, a aquellos escenarios que distinguen el nivel de albedo según la posición relativa dentro del cañón urbano -superficies horizontales: techos y pavimentos, -superficies verticales: fachadas-. Los niveles de reflectancia solar se dividen en: albedo bajo (â=0.3) y albedo alto (â=0.7) (ver rangos de albedo en sección 2.1 y Figura 4).

Figura 3. Validación del modelo numérico mediante regresión lineal de los datos medidos en la estación fija (Pf) y simulados (Ps). Fuente: Elaboración del autor. 


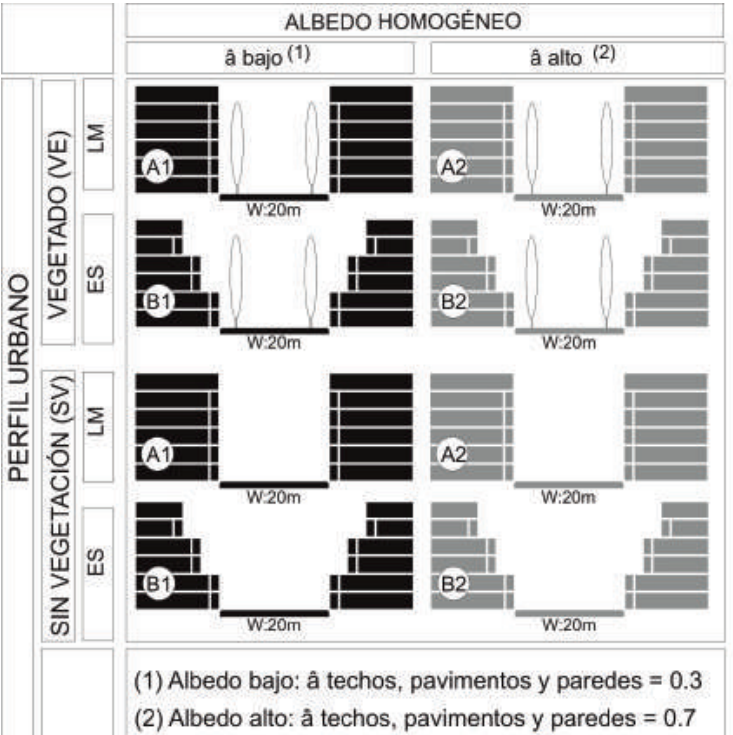

(2) Albedo alto: â techos, pavimentos y paredes $=0.7$
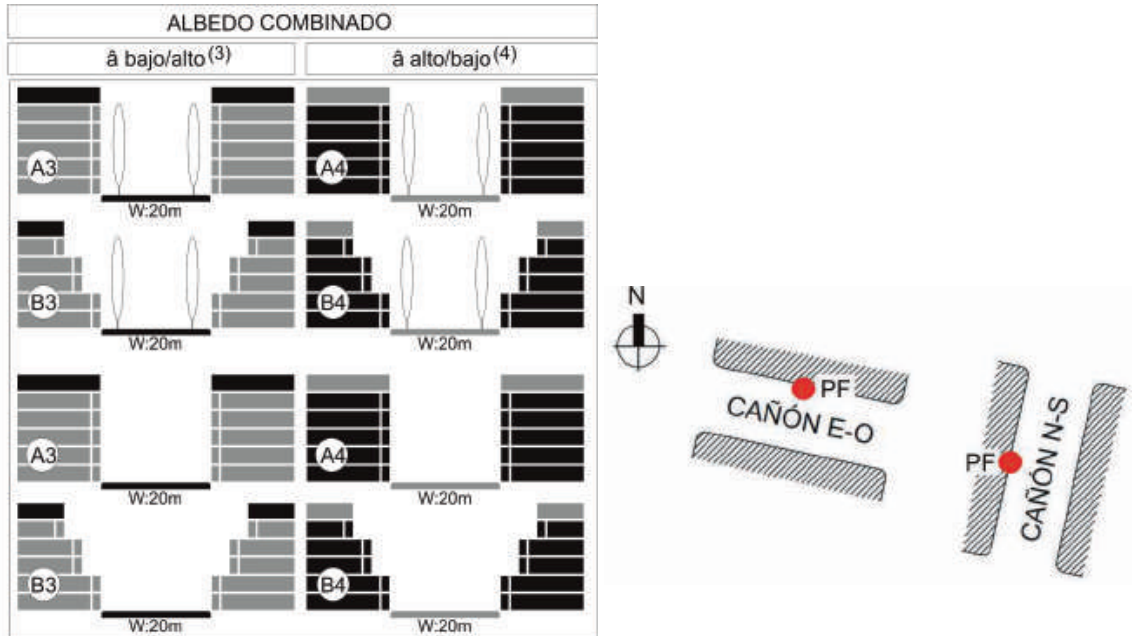

(3) Albedo bajo/alto: â techos y pavimentos = 0.3 ; â paredes $=0.7$

(4) Albedo alto/bajo: â techos y pavimentos =

$0.7 ;$ â paredes $=0.3$

Figura 4. Características de los escenarios propuestos según perfil urbano y nivel de albedo en envolventes. Fuente: Elaboración del autor.

Figura 5. Detalle de alternativas de orientación simuladas. Cañón vial Este- Oeste y cañón vial Norte -Sur. Fuente: Elaboración del autor.

Perfiles urbanos: Bajo un escenario de densificación máxima permitida por código (18 metros de altura), se diseñaron dos posibles configuraciones de perfiles urbanos: sobre línea municipal (A) y escalonado (B). El perfil A se eleva en forma de fachada continua. En cambio, en el perfil $B$, como su nombre lo indica, cada 6 metros de altura se produce un retiro de 3 metros respecto de la línea de edificación.

Porcentaje de vegetación: La vegetación es un componente determinante en el balance térmico de un recinto urbano, debido a que aporta sombra a las superficies expuestas y humedad al ambiente (Shishegar, 2014). Es por ello que se simularon dos configuraciones dentro del área a intervenir, la primera corresponde a un $60 \%$ de vegetación que coincide con el porcentaje y distribución de forestales del casode estudio (VE). La segunda configuración, sin vegetación (SV), fue estudiada para que el resto de los elementos no se vean enmascarados por el efecto del forestal (Figura 4).

Orientación: La habitabilidad del espacio público se determina en gran medida por la orientación de la sección de la calle, de modo que resulta fundamental considerar esta variable para analizar el nivel de asoleamiento que recibe cada canal vial. Fueron evaluadas dos orientaciones del canal vial:

- Este-Oeste (E-O): Orientación correspondiente al cañón vial donde se ubicó la estación fija de referencia (Pf), dentro del área de estudio. Rotación $12^{\circ}$ desde el norte.

- Norte- Sur (N-S): Se rotó el área de estudio $90^{\circ}$ desde el norte real, es decir, que en estos escenarios el cañón vial es perpendicular al actual. (Figura 5).

\section{RESULTADOS.}

\section{FACTOR DE VISIÓN DE CIELOSEGÚN MORFOLOGÍA EDILICIA/VEGETAL.}

La figura 6 grafica el factor de visión de cielo de las siguientes configuraciones: sin vegetación y perfil sobre línea municipal (SV_A); sin vegetación y perfil escalonado (SV_B); con vegetación y perfil sobre línea municipal (VE_A); y, con vegetación y perfil escalonado (VE_B).

En el total de las configuraciones evaluadas se relevó un factor de visión de cielo que oscila entre 0.28 y 0.66 . El mayor factor de visión a la bóveda celeste lo alcanzan aquellos escenarios sin vegetación y con perfil urbano escalonado (SV_B), indicados en la Figura 6.

\section{INTENSIDAD DE RADIACIÓN SOLAR SEGÚN MORFOLOGÍA EDILICIA/VEGETAL Y ORIENTACIÓN.}

La figura 7 grafica las curvas de radiación solar global (W/ $\mathrm{m}^{2}$ ) de las configuraciones que modifican su perfil urbano y porcentaje de vegetación.

Las curvas de radiación solar evidencian en las alternativas evaluadas que los escenarios vegetados disminuyen la intensidad hasta un $50 \%$ durante el mediodía solar.

Al comparar la orientación de los canales viales, se observa que la orientación N-S disminuye las horas en las que se encuentra irradiado un canal vial respecto a los cañones E-O. Además, en los cañones N-S la morfología del perfil 


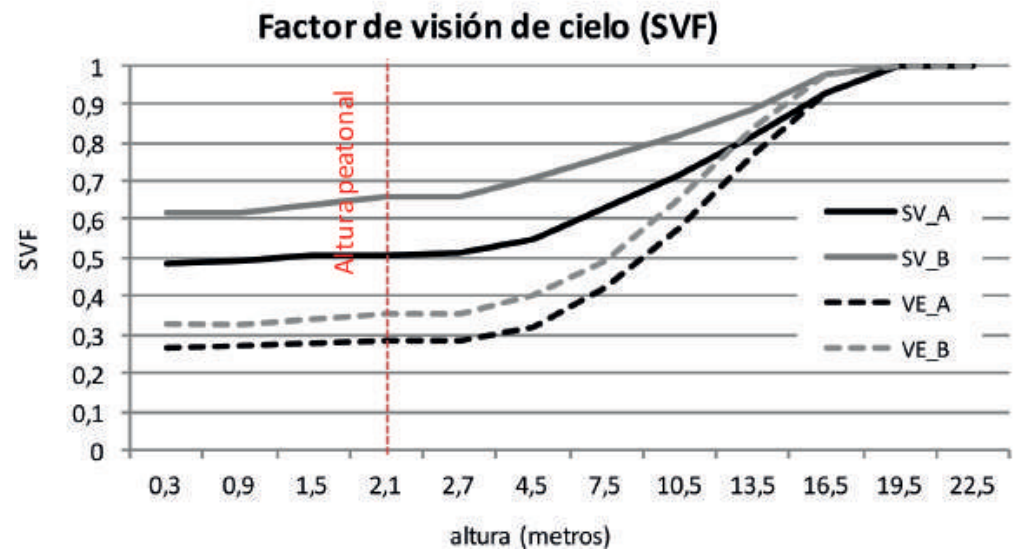

Figura 6. Factor de visión de cielo, según morfología edilicia y vegetal. Fuente: Elaboración del autor.
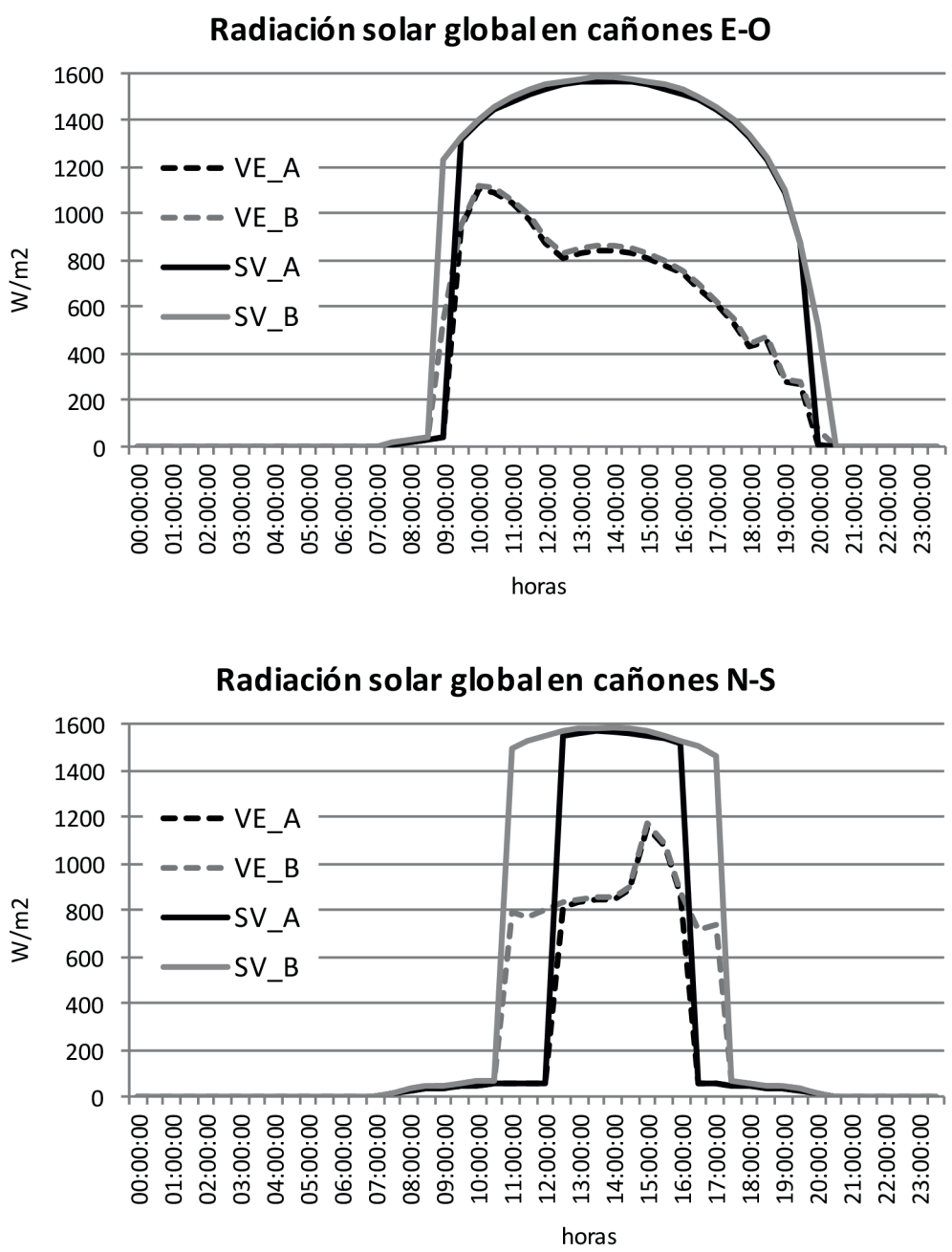

Figura 7. Intensidad de radiación solar global (W/m2) según morfología edilicia/ vegetal en cañones urbanos con orientación E-O (izquierda) y N-S (derecha). Fuente: Elaboración del autor. 


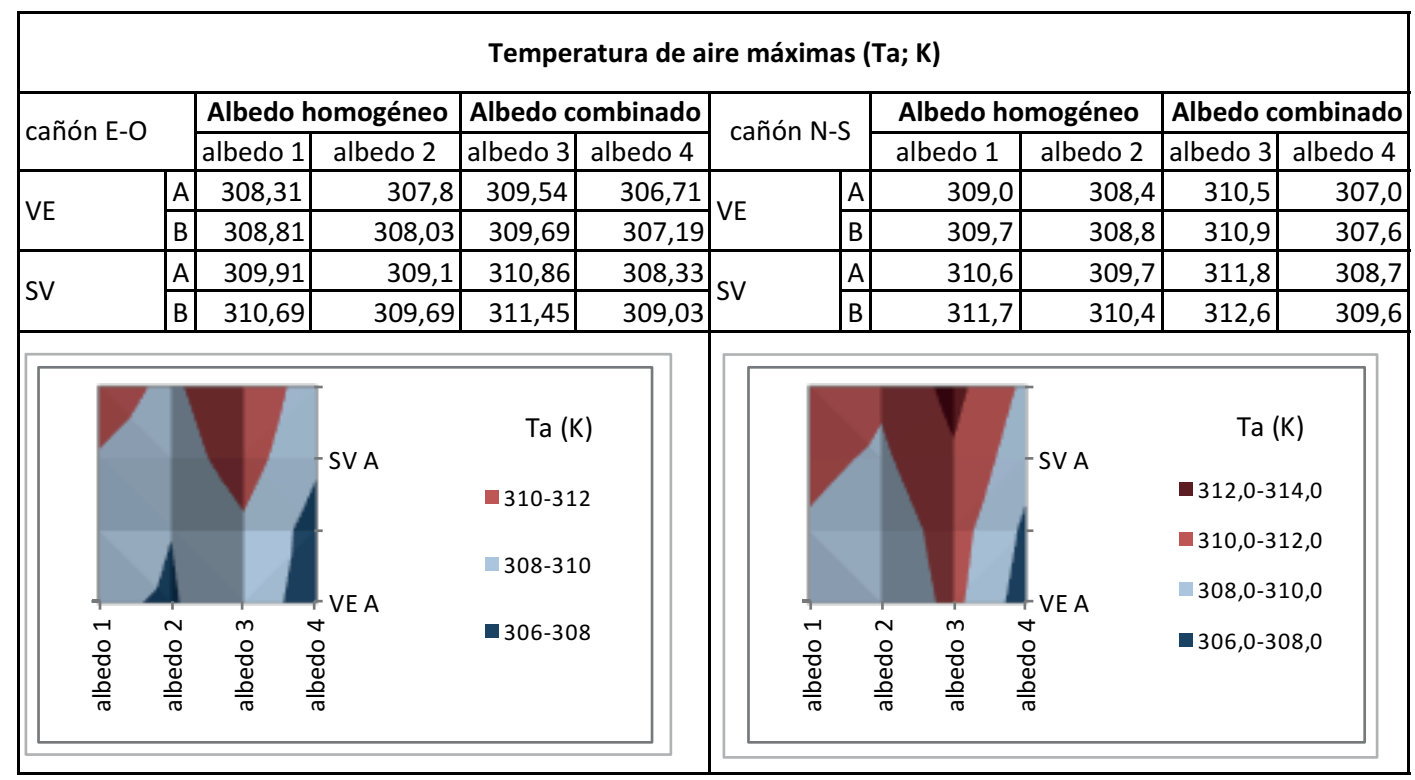

Tabla 3. Temperatura máxima de aire (Ta) según escenarios y orientación del cañón vial, a una altura de 2 metros y en grados Kelvin (K). Fuente: Elaboración del autor.

urbano es preponderante en lo referente al tiempo que permanece un canal vial irradiado. Los escenarios con perfil sobre la línea municipal (A) están en sombra 2 horas más durante el período de calentamiento y reducen 2 horas la insolación durante la tarde.

En suma, la vegetación (VE) disminuye la intensidad de radiación y la forma del perfil urbano $(\mathrm{A})$ reduce las horas de exposición, y de manera más significativa en la orientación N-S (Figura 7).

\section{TEMPERATURA DE AIRE MÁXIMA SEGÚN ESCENARIOS Y ORIENTACIÓN.}

La Tabla 3 describe las temperaturas máximas de aire, según escenario evaluado, a escala peatonal.

- Cañón vial E-O: Los escenarios más eficientes, es decir, los que menos elevan la Ta durante las horas de máxima radiación, son aquellos vegetados, que poseen albedo combinado rango 4, o sea, alto albedo sobre superficies horizontales y bajo albedo en superficies verticales, tanto para el perfil urbano sobre línea municipal (A) como para el escalonado (B). La alternativa con albedo homogéneo rango 2 , esto es, alto albedo en toda la envolvente, con vegetación y sobre línea municipal (A), también se mantiene más fría durante las máximas temperaturas (ver VE_ A4, VE_B4, y VE_A2 en Tabla 3).

Las alternativas más calientes corresponden a los escenarios sin vegetación, perfil escalonado (B) y con albedo rango 1 y 3 , es decir, las configuraciones que tienen niveles de albedo bajos en toda la envolvente y en las superficies horizontales respectivamente (ver SV_B1, SV_B3 y SV_A3 en Tabla 3).

- Cañón vial N-S: Al analizar las alternativas con cañones urbanos de orientación N-S, se evidencia que los escenarios más eficientes son aquellos vegetados, que poseen albedo combinado rango 4, para ambos perfiles urbanos (sobre línea municipal y escalonado) (ver VE_A4 y VE_B4 en Tabla 3).
La configuración que más eleva la Ta pertenece al escenario sin vegetación, con perfil escalonado y albedo combinado rango 3 , correspondiente a niveles de albedo bajo en techos y pavimento y niveles de albedo alto en fachadas, según SV_B3 en Tabla 3.

Al contrastar los comportamientos térmicos extremos entre escenarios, las diferencias de Ta registradas entre la configuración más fría (VE_A4) y la más caliente (SV_B3) son de $5^{\circ} \mathrm{K}$, en orientación de cañón E-O, y de $6^{\circ} \mathrm{K}$, en orientación N-S (Tabla 3).

Manteniendo constante la materialidad del canal vial y modificando la forma del perfil urbano (edilicia y vegetal), es posible disminuir la Ta hasta $2.4^{\circ} \mathrm{K}$ en canales viales $\mathrm{E}-\mathrm{O}$ y hasta $2.7^{\circ} \mathrm{K}$ en N-S.

Manteniendo constante la forma (edilicia y vegetal), las consecuencias de cambiar la materialidad del canal vial permiten disminuir la Ta hasta $2.8^{\circ} \mathrm{K}$ en E-O y hasta $3.5^{\circ} \mathrm{K}$ en N-S.

\section{TEMPERATURA MEDIA RADIANTE SEGÚN ESCENARIOS Y ORIENTACIÓN.}

La temperatura media radiante (Tmr) determina el grado de confort y nivel de habitabilidad de un espacio exterior. Es por ello, que en la Tabla 4 fueron detalladas, según configuración morfológica y material, a escala peatonal.

- Cañón vial E-O: Del total de los escenarios estudiados, aquellos que registran menor Tmr durante el periodo de calentamiento (8 a 20 h) están vegetados, tienen un rango de albedo homogéneo 1 (nivel de albedo bajo en toda la envolvente), tanto para el perfil urbano sobre línea municipal, como para el escalonado. Corresponden a los valores VE_A1 y VE_B1 en la Tabla 4.

Las alternativas que más elevan la Tmr no están vegetadas, tienen rangos de albedo 2, 3, y 4, para ambos perfiles urbanos, correspondiendo a los valores SV_A 2,3,4 y SV_B2, 3, 4 en la Tabla 4. 


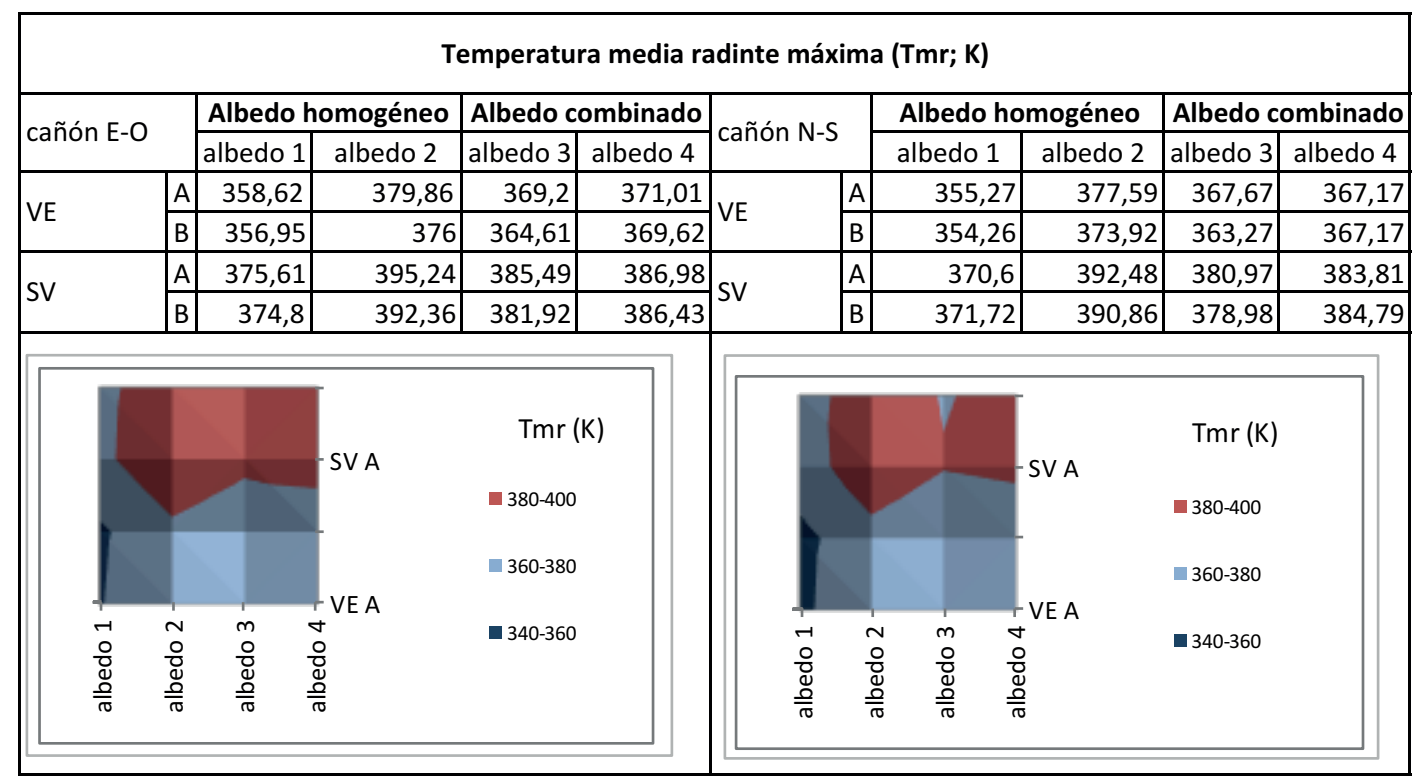

Tabla 4. Temperatura media radiante máxima (Tmr) según escenario y orientación del cañón vial, a una altura de 2 metros y en grados Kelvin (K). Fuente: Elaboración del autor.

- Cañón vial N-S: Al igual que en los cañones E-O, los escenarios con orientación N-S que elevan menos su Tmr están vegetados, tienen un rango de albedo 1 , con perfil urbano sobre línea municipal y escalonado, indicados en VE_A1 y VE_B1 en la Tabla 4.

Aquellos escenarios que más elevan la Tmr del canal vial N-S no están vegetados, tienen rangos de albedo 2,3 , y 4, para ambos perfiles urbanos como se indica en SV_A 2, 3,4 y SV_B 2, 4 en la Tabla 4.

Las Tmr extremas entre los escenarios evaluados, registran diferencias entre la configuración más fría (VE_A4) y la más caliente (SV_A2) igual a $24^{\circ} \mathrm{K}$ en orientación de cañón E-O y $25^{\circ} \mathrm{K}$ en orientación N-S (Tabla 4).

Manteniendo constante la materialidad del canal vial es posible disminuir la $\mathrm{Tmr}$ hasta $20.9^{\circ} \mathrm{K}$ en canales viales E-O, (VE_B3) y hasta $17.6^{\circ} \mathrm{K}$ (VE_B4) en escenarios vegetados y con perfil escalonado.

Manteniendo constante la forma (edilicia y vegetal), las consecuencias de cambiar la materialidad del canal vial permiten disminuir la Tmr hasta $21.2^{\circ} \mathrm{K}$ en $\mathrm{E}-\mathrm{O}$ y hasta $22.3^{\circ} \mathrm{K}$ en $\mathrm{N}-\mathrm{S}$, siendo más eficientes los escenarios con albedo homogéneo bajo (VE_A1).

Cabe destacar que los materiales para pavimentos que resultan más eficientes desde el punto de vista térmico, son aquellos con alto nivel de albedo, los cuales, sin embargo, pueden causar severos problemas de confort visual en un recinto urbano. Es por ello que una alternativa recomendable es la aplicación de pavimentos con textura, como es el caso del pavimento "Pétreo pulido circular gris-P13-", graficado en la Figura 1.

Anivelinternacional, investigaciones desarrolladas porSantamouris (2011) estudian superficies con geometría fractal -isotrópicas- que descomponen el haz de luz en todas las direcciones $y$, de esa forma, se disminuye el efecto de deslumbramiento trabajando sobre la rugosidad y texturas de las superficies o con pinturas termo crómicas que utilizan la energía de la radiación solar para cambiar de color.

\section{CONCLUSIONES.}

La morfología incrementa la captura de radiación solar y los materiales aumentan la permeabilidad, absortividad y capacidad calorífica de las superficies de la envolvente, provocando un crecimiento de las cargas térmicas dentro de un recinto urbano. El factor de visión de cielo (SVF) es un indicador morfo-forestal. El mismo relaciona la estructura morfológica edilicia y forestal de un canal urbano. En los escenarios analizados, a medida que el SVF se incrementa, también lo hacen la temperatura del aire $(\mathrm{Ta})$ y la temperatura media radiante (Tmr), debido al mayor ingreso de radiación de onda corta (solar) hacia el interior del cañón.

En un cañón urbano de 18 metros de alto y con un canal vial de 20 metros de ancho, la alternativa térmicamente más eficiente resulta de la configuración vegetada, con perfil urbano continuo sobre línea municipal y niveles de albedo alto en techos y pavimentos, y nivel de albedo bajo en fachadas. Estos escenarios registran disminuciones de $\operatorname{Ta}$ y $\mathrm{Tmr}$ de $6^{\circ}$ y $25^{\circ}$, respectivamente.

Los resultados revelan que incrementar el porcentaje de vegetación y aplicar materiales con altos niveles de albedo en techos y pavimentos, es la estrategia más efectiva para disminuir laTa.

Los parámetros de diseño que impactan en mayor medida sobre la reducción de la Tmr comprenden el incremento de la vegetación dentro del canal vial y la incorporación de materiales para fachadas con bajo nivel de albedo. La modificación de estas variables tiene un efecto más notorio en canales viales con orientación N-S que en aquellos E-O. Cabe subrayar que la densificación conlleva al incremento de la Tmr y éste, a su vez, produce la disminución de la habitabilidad térmica de los canales viales.

Al contrastar el peso de cada variable, se evidencia que la modificación del material de la envolvente tiene mayor impacto que la modificación de la morfología edilicia, tanto en canales vegetados, como sin vegetar. Esta información permite 
rehabilitar térmicamente un recinto urbano mediante el empleo de materiales que se adecuen a las necesidades particulares de cada espacio, de acuerdo a su morfología, clima y orientación, sin requerir de una intervención de mayor envergadura que incluya trabajar sobre la forma edilicia.

Este trabajo es de carácter inicial, no obstante, entre las medidas de diseño identificadas se encuentran la promoción de materiales de construcción con alto albedo sobre superficies horizontales, formas de diseño del hábitat que disminuyan el periodo diario de exposición a la radiación solar -como es el caso del perfil urbano sobre línea municipal- y los trazados urbanos que faciliten los procesos de renovación atmosférica -orientación que favorezca la ventilación urbana. Estos criterios, aplicados al planeamiento urbano, contribuirán, en definitiva, a mejorar la eficiencia energética y ambiental en ciudades con altos niveles de radiación solar.

\section{AGRADECIMIENTOS.}

Se agradece a la Agencia Nacional de Promoción Científica y Tecnológica -ANPCYT- y al Consejo Nacional de Investigaciones Científicas y Técnicas -CONICET- que proporcionaron los fondos para la presente investigación.

\section{REFERENCIAS BIBLIOGRÁFICAS.}

AKBARI, Hashem; LEVINSON, Ronnen y STERNB, Stephanie. Procedure for measuring the solar reflectance of flat or curved roofing assemblies. Solar Energy, 2008, vol. 82, n7, pp. 648-655. AKBARI, Hashem; MENON, Surabi y ROSENFELD, Arthur. Global cooling: increasing world-wide urban albedos to offset CO2. Climatic Change, 2009, vol. 94, n³, pp. 275-286.

ALCHAPAR, Noelia; CORREA, Érica y CANTÓN, M. Alicia. Classification of building materials used in the urban envelopes according to their capacity for mitigation of the urban heat island in semiarid zones. Energy and Buildings, 2014, n69, pp. 22-32.

ALCHAPAR, Noelia y CORREA, Érica. Aging of roof coatings. solar reflectance stability according to their morphological characteristics. Construction and Building Materials, 2015, n 102 , pp. 297-305.

ALI-TOUDERT, Fazia y MAYER, Helmut. Numerical study on the effects of aspect ratio and orientation on an urban street canyon on outdoor thermal comfort in hot and dry climate. Building and Environmet, 2006, n41, pp. 94-108.

BRUSE, Michael. ENVI-met 3.1. On-Line Manual [en línea], 2006. [Consultado 22 mayo 2016] Disponible en: http://www.envi-met. com.

CORREA, Érica. Isla de Calor Urbana. El caso del área metropolitana de Mendoza. Tesis doctoral inédita, Universidad Nacional de Salta, Argentina, 2006.

CORREA, Érica; RUIZ, M. Angélica y CANTÓN M. Alicia. Morfología forestal y confort térmico en "ciudades oasis" de zonas áridas. Ambiente Construido, 2010, vol. 10, n4, pp. 119 $-137$.
DOYA, Maxime; BOZONNET, Emmanuel y ALLARD Francis. Experimental measurement of cool facades performance in a dense urban environment. Energy and Buildings, 2012, n 55, pp. 42-50.

ERELL, Evyatar; PEARLMUTTER, David; BONEH, Daniel y KUTIEL, Pua Bar. Effect of high-albedo materials on pedestrian heat stress in urban canyons. Urban Climate, 2014, n ${ }^{\circ} 0$, pp. 367-386.

GIVONI, Baruch; Noguchi, MIKIKO; SAARONI, Hadas; POCHTER, Oded; YAACOV, Yaron; FELLER, Noa y BECKER, Stefan. Outdoor comfort research issues. Energy and Buildings, 2003, vol. 35, $\mathrm{n}^{\circ} 1$, pp. 77-86

JOHANSSON, Erik y EMMANUEL, Rohinton. The influence of urban design on outdoor thermal comfort in the hot, humid city of Colombo, Sri Lanka. International Journal Biometeorology, 2006, vol. 51, n², pp. 119-133.

KOTTEK, Markus; GRIESER, Jürgen; BECK, Christoph; RUDOLF, Bruno y RUBEL, Franz. World Map of the Köppen-Geiger climate classification updated. Meteorologische Zeitschrift, 2006, vol. 15, $n^{\circ} 3$, pp. 259-263.

PEARLMUTTER, David; BERLINER, P., y SHAVIV, E. Urban climatology in arid regions: current research in the Negev desert. International Journal of Climatology, 2007, vol. 27, n¹4, pp.18751885.

SANTAMOURIS, Matheos. Advances in passive cooling (Buildings, energy and solar technology series). Londres: Earthscan Publications Ltd., 2007.

SANTAMOURIS, Matheos. Energy and climate in the urban built environment. New York: Routledge Publications. Taylor \& Francis Group, 2011

SANTAMOURIS Matheos. Cooling the cities - A review of reflective and green roof mitigation technologies to fight heat island and improve comfort in urban environments. Solar Energy, 2014, n¹03, pp. 682-703.

SHASHUA-BAR, Limor; TSIROS, loannis y HOFFMAN, Milo. Passive cooling design options to ameliorate thermal comfort in urban streets of a Mediterranean climate (Athens) under hot summer conditions. Building and Environment, 2012, $n^{\circ} 57$, pp.110-119.

SHISHEGAR, Nastaran. The Impact of Green Areas on Mitigating Urban Heat Island Effect: A Review. The International Journal of Environmental Sustainability, 2014, vol. 9, n¹, pp. 119-130.

THORSSON, Sofia; LINDBERG, Fredrik; BJÖRKLUND, Jesper; HOLMER, Björn yRAYNER, David. Potential changes in outdoor thermal comfort conditions in Gothenburg, Sweden due to climate change: the influence of urban geometry. International Journal of Climatology, 2010, vol. 31, n², pp. 324-335.

YAGHOOBIAN, Neda y KLEISSL, Jan. Effect of reflective pavements on building energy use. Urban Climate, 2012, vol. 2, pp. 25-42. 